\title{
Using Personas to Model Museum Visitors
}

\author{
Moneerah Almeshari \\ Computer Science \\ University College London \\ London, United Kingdom \\ moneerah.almeshari.17@ucl.ac.uk
}

\author{
John Dowell \\ Computer Science \\ University College London \\ London, United Kingdom \\ j.dowell@ucl.ac.uk
}

\author{
Julianne Nyhan \\ Information Studies \\ University College London \\ London, United Kingdom \\ j.nyhan@ucl.ac.uk
}

\begin{abstract}
It is widely agreed that museums and other cultural heritage venues should provide visitors with personalised interaction and services such as personalised mobile guides, although currently most do not. Since museum visitors are typically first-time visitors and since their visit is for a relatively short session, personalisation should use initial interaction data to associate the user with a particular persona and thereby infer other facts about the user's preferences and needs. In this paper we report a questionnaire-based study carried out with 105 visitors of a Science and Technology Centre to examine the minimal features needed to identify visitor personas. We find that museum visitors can be clustered by their visit motivation and perceived success factors; these clusters are found to correspond both with Falk's visitor categorisation and a prior classification of exploration styles. Consequently, these two features can be used to reliably identify the visitor persona, and therefore, can be used for user modeling.
\end{abstract}

\section{KEYWORDS}

Museum visitors, personas, user modeling, mobile guides.

\section{ACM Reference format:}

Moneerah Almeshari, John Dowell and Julianne Nyhan. 2019. Using Personas to Model Museum Visitors. In Proceedings of ACM 27th Conference on User Modeling, Adaptation and Personalization Adjunct (UMAP'19 Adjunct). ACM, Larnaca, Cyprus, ACM, New York, NY, USA, 5 pages. https://doi.org/10.1145/3314183.3323867

\section{Introduction}

A consensus has emerged amongst researchers and developers that personalisation is a priority requirement for mobile guides (MGs) in museums and cultural heritage sites [16]. Visitors are extremely varied in terms of their motivations, goals and needs, knowledge of the venue and their interest in knowing and experiencing more [7]. Responding to these individual differences requires guides to personalise the user interface (UI) and content rather than simply offering the same choices to all users [8].

Permission to make digital or hard copies of all or part of this work for personal or classroom use is granted without fee provided that copies are not made or distributed for profit or commercial advantage and that copies bear this notice and the full citation on the first page. Copyrights for components of this work owned by others than ACM must be honored. Abstracting with credit is permitted. To copy otherwise, or republish, to post on servers or to redistribute to lists, requires prior specific permission and/or a fee. Request permissions from Permissions@acm.org.

UMAP'19 Adjunct, fune 9-12, 2019, Larnaca, Cyprus

(C) 2019 Association for Computing Machinery.

ACM ISBN 978-1-4503-6711-0/19/06...\$15.00

https://doi.org/10.1145/3314183.3323867
Personalisation relies on a user model which is defined as "a data structure that represents user interests, goals and behaviours" [9]. Consequently, the quality of the user model has a direct impact on the quality of the personalisation.

The 'cold start problem' occurs where the system does not have prior information about the user and needs to construct the user model from scratch during their first session. This is a formidable challenge in settings like museums where visitors are mostly first time visitors and their usage is for a relatively short session [10]. A promising response to this involves the use of initial data to associate a user with a particular user category and thereby model the user or infer other facts about their preferences and needs [10]. This approach places a user in a category based on certain parameters and adapts the UI accordingly. A number of adaptive cultural heritage projects have used this method such as AVANTI [11], PEACH [12], PeVEP [13] and INTRIGUE [14].

Visitors to museums have been extensively studied and several visitor taxonomies have been identified. We refer to such a visitor category as a persona, representing "an aggregate of target users who share common behavioural characteristics" [15]. Personas assist in focusing on some aspects of how a group of people think, behave, communicate, what they need to achieve and why [16]. A persona represents a particular type of interactive product user [16]; very importantly, it identifies the particular motivation and goals that determine the characteristic interactive behaviour of a persona [16]. Amongst the abundance of tools available for interaction design, personas have been claimed to be "the most effective and fundamental" [16]. By creating a concrete image of the user, designers gain insight into the elements a UI should have and how a target user population is likely to respond to those elements [16].

Personas may be reified by presenting them as fictional characters with a name, gender, ethnicity, and even the kind of car they drive [19]. However Cooper, Reimann [19] persuasively argue that personas are fundamentally about motivations and goals rather than tasks and demographics. Researchers have focused on modelling museum visitors in relation to personalisation using complicated features like visiting style and simple features like age and location, while neglecting crucial dimensions like visit motivation [8]. In this paper we focus on the most important factors that determine a persona: motivation, behaviour and goals.

The study exploits Falk's taxonomy of six types of museum visitor (i.e., personas) [17]. This robust taxonomy has been 
chosen because it has been widely used and tested in museums and Science Centres. Moreover, the individual does not have to be physically present in the museum to supply the required information, so the process does not necessarily impinge on their actual museum visit.

There are two main aspects to determine when using this approach for user modeling. First: what is the minimal set of defining features that can reliably be used to distinguish a user's persona and infer information about them? Second: what are the common preferences and attributes of those personas and how do they guide the personalisation process?

In this paper we investigate sources and forms of evidence needed to model museum visitors as belonging to particular persona types based on Falk's classification [17] using a questionnaire. We examine whether the user model generated by the questionnaire corresponds with the visitor's self-assessment of their persona type to determine the questionnaire accuracy in identifying the visitor persona. In addition, we examine the association between different personas and their self-assessed exploration style characterised by [18]. Knowing such information can assist in modeling visitor personas implicitly.

\section{Related Work}

Personalisation can be implemented using implicit or explicit approaches [10]. In the implicit approach, the user profile is built by inference from data about the user's behaviour including their choice. By contrast, the explicit approach builds the user profile by asking the user for information either directly or indirectly. The direct explicit approach asks users about their preferences and needs which, in the indirect approach, are inferred from answers to tangential questions about different domain. The explicit approach is desirable when a user model must be constructed rapidly in environments such as museums where time is limited. Antoniou, Katifori [10] used a short questionnaire to elicit visitor characteristics, preferences and visiting contexts using indirect questions to provide personalised interactive storytelling at a museum.

As discussed earlier, minimal gathered data can be used to place the user in a category to infer more facts needed for personalisation. Various categorisations of museum visitors have been identified based on different constructs. Differences in the way museum visitors prefer to learn have been characterised with some preferring 'mind-on' and hands-on activities, and others preferring to explore and reflect [19]. Differences in expertise have also been used to differentiate museum visitors [20, 21]. In addition, visitors' behaviour in navigating between exhibits within a venue has been categorised as four distinct types [18].

Falk [17] categorised visitors on the basis of their visit motivation which is seen as shaping both the expectation and experience of the visit. He identifies five visitor personas: Explorers, Facilitators, Experience seekers, Professionals/Hobbyists; and Rechargers [17]. Explorers visit to satisfy their curiosity and have a general interest in the museum [17]. Facilitators visit to support other people they care about such as kids or companions; the experience of these others directly affects the facilitators' own experience [17]. Experience seekers visit because they are conscious of the reputation of the venue; they visit more for pleasure than for knowledge [17]. Professionals/ Hobbyists visit because of particular interests and they have clear objectives for their visit [17]. Rechargers visit seeking contemplative, spiritual and restorative experiences, hoping to relax and recharge their energies [17]. To identify the visitor persona, Falk has used interviews and questionnaires. A 20 element questionnaire suitable for visitors at a zoo and aquarium has been used in [22] to study the relationship between these identity-related motivations and their visit conservation learning.

\section{Method}

A face to face questionnaire was deployed over four weeks in 2018 in Scitech, a visitor centre housing exhibits of science and technology in Saudi Arabia. The questionnaire had been reviewed by 4 museum curators. Arabic and English versions of the questionnaire were created and assessed for accuracy by 2 Arab specialists of English language. The questionnaire was pilot tested with 5 museum visitors before gathering the main data.

Individuals were invited to complete the questionnaire at the end of their visit. The questionnaire asked visitors about their demographics and visit. It included a set of multiple-choice questions based on the five factors that characterise visitor personas in Falk's description. The five factors are visit motivation, visit success criteria, social aspects, knowledge of museum content, and number of visits. In addition, the questionnaire asked participants to identify their exploration style as one of the following [18]:

a. I see almost all objects and read object labels in detail

b. I explore and I avoid looking at objects' information in detail

c. I explore and I stop to see details of some objects that attract me

d. I spent my time visiting specific exhibitions or objects "planned before the visit" and ignoring the others

Visitors were then shown five visitor persona descriptions (Explorer, etc) and asked to identify themselves with one. This self-assessment provides a ground-truth reference for determining the questionnaire's accuracy in identifying a visitor's persona.

105 visitors participated in the study of whom 66 were female, 65 adults, 34 teenagers, 3 seniors and 3 were children. It was the first visit to Scitech for 45 participants, 39 participants had visited 2 to 4 times previously and 21 had visited 5 times or more.

SPSS was used to analyse the collected data which required Chi Square tests as it is entirely categorical. Cramer's V test was used to indicate the strength of the relationship where a significant effect was found.

\section{Eliciting Visitor Personas}

In the persona self-assessment question, 33 visitors identified themselves as Facilitators (31.4\%), 29 as Explorers (27.6\%), 18 as 
Experience Seekers (17.1\%), 13 as Professionals/Hobbyists (12.4\%) and 12 as Rechargers (11.4\%).

\subsection{Main Categorisation Factors}

Significant correlations are found between the self-assessed persona type and the four factors of: motivation $\chi 2(24, \mathrm{~N}=105)$ $=90.274, \mathrm{p}=0.000<$ alpha $=0.05 ;$ success criteria $\chi 2(20, \mathrm{~N}=105)$ $=79.076, \mathrm{p}=0.000$; social aspect $\chi 2(28, \mathrm{~N}=105)=52.277, \mathrm{p}=0.01$; and number of visits $\chi 2(8, N=105)=23.399, p=0.003$. No significant difference between visitor types was found in relation to knowledge of museum content $\chi 2(16, \mathrm{~N}=105)=25.103, \mathrm{p}$ $=0.068$.

TwoStep clustering was employed to classify respondents using the four significant factors, however, it resulted in a poor silhouette measure (0.2) for cohesion and separation of the clusters. Examining the predictor importance index values, it was found that social aspect and number of prior visits were the least important factors in the clustering.

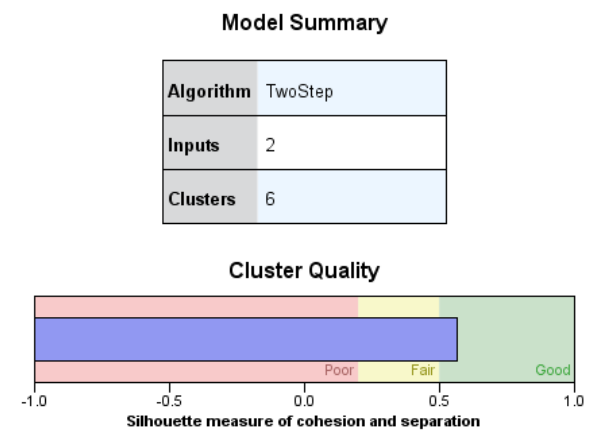

Figure 1: TwoStep clustering quality indicator with Motivation and Success Criteria

Re-running the TwoStep clustering with just the two primary factors of motivation and success criteria produced 6 clusters with a good silhouette measure (0.6) of cohesion and separation (Figure 1). The question that elicits visitor motivation is "What motivated you to visit Scitech today?". For success criteria it is "Which of the following means that your visit was successful, and that you achieved what you aimed for?"

\subsection{Persona Identification Accuracy and Parameters}

We then needed to establish whether these six clusters produced by the algorithm using motivation and success criteria factors can be associated with the five visitor personas derived from Falk [19] (when additionally considering the Facilitator subtypes of Parental Facilitator and Socializer Facilitator). Examining the majority class of response in each cluster in relation to the two primary factors of Motivation and Success Criteria, we found each cluster can indeed be identified uniquely with a particular persona.

\begin{tabular}{c|cc} 
Visitor Persona & $\begin{array}{c}\text { Correctly } \\
\text { Assigned }\end{array}$ & $\begin{array}{c}\text { Incorrectly } \\
\text { Assigned }\end{array}$ \\
\hline Facilitator & 33 & 0 \\
Explorer & 21 & 8 \\
Experience Seeker & 17 & 1 \\
Professional/Hobbyist & 13 & 0 \\
Recharger & 11 & 1
\end{tabular}

Table 1: Accuracy of the clustering method in assigning visitor persona; ground truth is visitors' self-assignment.

A Chi square test indicated a significant association between the self-assessed persona and cluster assigned persona $\chi 2(20$, $\mathrm{N}=105)=83.108, \mathrm{p}=0.000 .90 .5 \%$ of self-assessed personas have been identified correctly using the questionnaire (Table1). $100 \%$ of Facilitators and Professional/Hobbyists, $94 \%$ of Experience Seekers, $92 \%$ of Rechargers and $72 \%$ of Explorers have been assigned to the persona accurately using the clusters produced by the two questions. In other words, the persona that a visitor associated themselves with reliably coincided with the persona type found by clustering their answers to the questionnaire.

The answers of the two questions of each persona (cluster) are mapped in Figure 2. The mapping was based on the clustering results where it has assigned each cluster with the most common answers to the two factors (Table 2).

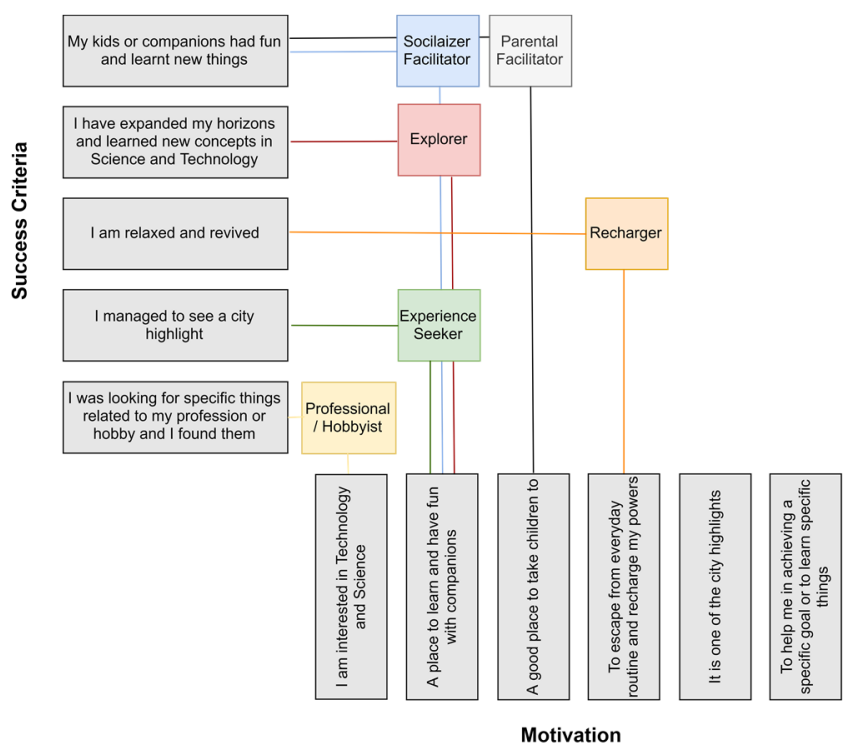

Figure 2: Assigned visitor personas and their relation to the visit motivation and the perceived success criteria

Figure 2 shows that the same motivation of our visitor participant is found with three different personas who all indicated "a place to learn and have fun with companions". Explorers and Experience Seekers can come alone to the museum, so this answer cannot be used to uniquely identify the visitor persona. On the other hand, results indicate that the success criterion question alone is able to identify the five visitor 
personas. However, facilitator subtypes cannot be identified unless the motivation is also indicated.

\begin{tabular}{c|cc} 
Visitor Persona & Success Criteria & Motivation \\
\hline Parental Facilitator & $\% 100$ & $\% 100$ \\
Socializer Facilitator & $\% 100$ & $\% 57$ \\
Explorer & $\% 100$ & $\% 48$ \\
Experience Seeker & $\% 53$ & $\% 71$ \\
Professional/Hobbyist & $\% 46$ & $\% 46$ \\
Recharger & $\% 100$ & $\% 100$
\end{tabular}

\section{Table 2: Persona proportions used for the mapping} presented in figure 2

Taken together, these results show first, that the questionnaire is able to identify museum visitors' self-assessed personas as they relate to Falk's visitor types: answers to the separate questions correlate with participants' self-assessments. Second, clustering answers to the primary questionnaire factors produces a set of clusters that correspond with participants' selfassessments. This means that users of a MG can be asked the two questions about their motivation and success criteria and the MG will be able to reliably determine their visitor persona within the Falk taxonomy of identity-related motivations and consequently generate the user model.

\section{Exploration Style}

Participants were asked about their museum exploration style to find if it is associated with their visitor persona. They were asked to choose between four descriptions of exploration style, labelled as Ant, Fish, Butterfly and Grasshopper [18]. Ants are visitors who view almost all exhibits and follow a definite path close to exhibits; Fish are visitors who avoid detailed exhibit information and proximity to exhibits, preferring instead to view at a distance from open spaces; Butterflies change direction frequently and view most exhibits in detail but for varying times; Grasshoppers view specific exhibits only (often planned before the visit), spending time looking at them in detail and ignoring other exhibits [23].

A significant association between visitor persona and selfassessed exploration style has been found $\chi 2(15, \mathrm{~N}=105), \mathrm{p}=0.015$ with a moderately strong relationship $(\mathrm{V}=0.273)>0.20$. Results indicate that visitor personas are divided into two groups based on their self-identified exploration style. The greatest percentage of Facilitators (both parental 59\% and socializers 48\%) indicated that they view almost all objects and read objects details (Ants). The other four personas indicated that they explore and stop to see details of some objects that attract them (Butterflies), including the majority of Rechargers (79\%) and Professionals/Hobbyists (69\%) and the largest percentage of Explorers (48\%) and Experience Seekers (41\%) (Table 3).

\begin{tabular}{c|cccc} 
Visitor Persona & Ant & Butterfly & Fish & Grasshopper \\
\hline Parental Facilitator & $\mathbf{1 3}$ & 8 & 1 & $\mathbf{0}$ \\
Socializer Facilitator & $\mathbf{1 0}$ & 7 & 3 & 1 \\
Recharger & $\mathbf{0}$ & $\mathbf{8}$ & 3 & $\mathbf{0}$ \\
Professional/Hobbyist & 3 & $\mathbf{9}$ & $\mathbf{0}$ & 1 \\
Explorer & 7 & $\mathbf{1 0}$ & 3 & 1 \\
Experience Seeker & 4 & $\mathbf{7}$ & 4 & 2
\end{tabular}

Table 3: Visitor personas and their self-assessed exploration style corresponding to [18] classification

In addition, table 3 shows that none of the Professional/Hobbyist visitors have assessed their exploration style as Fish, no Rechargers have assessed themselves as Ant and no Rechargers or Parental Facilitators have assessed their exploration style as Grasshopper.

Knowing the exploration style of different visitor personas offers the opportunity to generate the user model implicitly by recognizing individual visitor's patterns of viewing exhibits. However, since our results are based on self-assessment, they would benefit from further validation with observational methods.

\section{Discussion}

Personas are a widely used and highly valued technique in interface design practice[16]. Our study has used the concept of personas to model users into distinct sub-populations of museum visitors. We developed a questionnaire for museum visitors that can associate individuals with particular personas.

Our study found that visitors can be clustered into distinct types correspond with Falk's identity related motivation categories using two minimal trigger features: motivation and visit success factors. We used a set of persona definitions produced by Falk's studies of museum visitors to validate the categorisation and we showed that answers to the two questions about motivation and perceived success criteria would place people in the same category as they would place themselves.

We explored how different visitor personas assess their exploration style based on the classification of [18]. The most common exploration style for each persona group of participants was either Ant or Butterfly. This was against expectation for Professional/Hobbyists who typically are looking for specific exhibits and would therefore be more likely to self-identify as Grasshoppers. On the other hand, no Rechargers assessed themselves as either Ants or Grasshoppers and this is consistent with their description and needs. Knowing the exploration style of each persona can assist in detecting them automatically using their movement patterns to provide adaptive services such as adaptive MGs.

In conclusion, our study indicates that a MG would be able to generate a user model from a 'cold start' (i.e., with no other information) simply by asking the two questions about the user's motivation in visiting the museum and how they would judge the success of their visit. 


\section{REFERENCES}

[1] Kabassi, K., Personalisation systems for cultural tourism, in Multimedia services in intelligent environments. 2013, Springer. p. 101-111.

[2] Ardissono, L., T. Kuflik, and D. Petrelli, Personalization in cultural heritage: the road travelled and the one ahead. User modeling and user-adapted interaction, 2012. 22(1-2): p. 73-99.

[3] Fidas, C.A. and N.M. Avouris. Personalization of mobile applications in cultural heritage environments. in IISA. 2015.

[4] Goren-Bar, D., et al., I like it-An Affective Interface for a Multimodal Museum Guide. Proceedings of Intelligent User Interfaces IUI'05, 2005.

[5] Not, E. and D. Petrelli. Balancing Adaptivity and Customisation. in International Conference on User Modeling, Adaptation, and Personalization. 2014. Springer.

[6] Fosh, L., et al. Gifting as a Novel Mechanism for Personalized Museum and Gallery Interpretation. in International Conference on User Modeling, Adaptation, and Personalization. 2015. Springer.

[7] Falk, J.H. and L.D. Dierking, The museum experience revisited. 2016: Routledge.

[8] Emmanouilidis, C., R.-A. Koutsiamanis, and A. Tasidou, Mobile guides: Taxonomy of architectures, context awareness, technologies and applications. Journal of network and computer applications, 2013. 36(1): p. 103125.

[9] Frias-Martinez, E., et al., Automated user modeling for personalized digital libraries. International Journal of Information Management, 2006. 26(3): p. 234-248.

[10] Antoniou, A., et al. Capturing the visitor profile for a personalized mobile museum experience: an indirect approach. in UMAP (Extended Proceedings). 2016. Halifax, Canada.

[11] Fink, J., A. Kobsa, and A. Nill, Adaptable and adaptive information provision for all users, including disabled and elderly people. New review of Hypermedia and Multimedia, 1998. 4(1): p. 163-188.
[12] Stock, O., et al., Adaptive, intelligent presentation of information for the museum visitor in PEACH. User Modeling and User-Adapted Interaction, 2007. 17(3): p. 257-304.

[13] Bonis, B., et al., A platform for virtual museums with personalized content. Multimedia tools and applications, 2009. 42(2): p. 139-159.

[14] Ardissono, L., et al., Intrigue: personalized recommenation of tourist attractions for desktop and hand held devices. Applied artificial intelligence, 2003. 17(8-9): p. 687-714.

[15] Miaskiewicz, T. and K.A. Kozar, Personas and usercentered dsign: How can personas benefit product design processes? Design Studies, 2011. 32(5): p. 417-430.

[16] Cooper, A., et al., About face: the essentials of interaction design. 2014: John Wiley \& Sons.

[17] Falk, J.H., Identity and the museum visitor experience. 2016: Routedge.

[18] Véron, E. and M. Levasseur, Ethnographie de l'expositio: l'espace, le corps et le sens. 1989: Centre Georges Pompidou, Bibliothèque publique d'information.

[19] Luyten, K., et al. ARCHIE: disclosing a museum by a socially-aware mobile guide. in 7 th International Symposium on Virtual Reality, Archaeology and Cultural Heritage. 2006.

[20] Walsh, D., P. Clough, and J. Foster, User Categories for Digital Cultural Heritage. 2016.

[21] Falk, J.H. and L.D. Dierking, Learning from museums: Visitor experiences and the making of meaning. 2000: Altamira Press.

[22] Falk, J.H., J. Heimlich, and K. Bronnenkant, Using identity-related visit motivations as a tool for understanding adult zoo and aquarium visitors' meaning-making. Curator: The Museum Journal, 2008. 51(1): p. 55-79.

[23] Zancanaro, M., et al. Analyzing museum visitors' behavior patterns. in International Conference on User Modeling. 2007. https://www.researchgate.net/profile/Zvi Boger/public ation/221260782_Analyzing_Museum_Visitors'_Behavi or Patterns/links/0fcfd505758b80066e000000.pdf: Springer. 